\title{
Using power-consuming materials for volume damping of thin-walled structural elements
}

\author{
Vladimir Mironov ${ }^{1,2}$, Dmitriy Ogorelkov ${ }^{2,}$ and Olga Lukashuk ${ }^{1}$ \\ ${ }^{1}$ Ural Federal University, 19 Mira street, Ekaterinburg, 620002, Russia \\ ${ }^{2}$ Institute of Engineering Science, Ural Branch of the Russian Academy of Sciences, 34 \\ Komsomolskaya street, Ekaterinburg, Russia
}

\begin{abstract}
Using low-modulus foam materials of lower density leads to significantly higher protection of personnel (from noise and harmful vibrations) and structures (from corrosion), while only slightly increasing the weight of an object. But the main intended purpose of such a filler which is capable of actively absorbing the energy of vibrations caused by external dynamic forces is to reduce their number and amplitude. To achieve a similar effect, the building industry and aircraft construction have a long and successful history of utilizing three-layer plates and shells coated with a thin foam material. Theoretical studies, supported by experiments, make one come to the conclusion that volume damping of such elements has quite a low efficiency. The goal of the research was to preliminarily estimate how appropriate it is to use artificial low-modulus elastic materials to damp thin-walled elements of metal structures. It was achieved by running computational experiments in Solid Works software environment and plotting, on the basis of the experiments, vibrograms of damped vibrations of a cantilever beam. The results obtained during the testing showed that the volume damping using a foam material could be an efficient tool for damping free vibrations of a metal structure affected by bending.
\end{abstract}

\section{Introduction}

Dissipation of energy in a material is caused by its elastic imperfections and manifests itself in the formation of a certain hysteresis loop under cyclic strain [1]. Regardless of what causes the energy losses, damping properties of an elastic system are thought to be characterized by a relative dissipation of energy $\psi$, which is considered in terms of a relation between a dissipated energy $\Delta W$ during one cycle of steady-state vibration and an amplitude value of a potential energy $W$ in the elastic system [1-3]:

$$
\psi=\frac{\Delta W}{W}
$$

\footnotetext{
*Corresponding author: ogorelkov.dimon@yandex.ru
} 
The relative energy dissipation $\psi$, expressed by (1) and often called a coefficient of dissipation or absorption, is utilized to estimate the damping properties of a material. The absorption coefficient of a structure could be calculated only for the simplest cases. An expression which describes the absorption coefficient $\psi_{R}$ of a three-layer coated rod under pure bending is known [2]:

$$
\psi_{R}=\psi+\psi_{A} \frac{6 E_{A} V_{A}}{E V}
$$

where $\psi, \psi_{A}$ are absorption coefficients for an uncoated rod and a coating material, $V$, $V_{A}$ and $E, E_{A}$ are volumes and moduli of elasticity for the rod and coating material. It was noted that the absorption-coefficient correction for a coated rod defined by the second summand depends on the product of $\psi_{A} E_{A}$. Therefore, coatings made of low-modulus materials with quite high damping properties often turn out to be inefficient $[4,5]$.

\section{Theoretical estimation of the absorption coefficient of a filled beam}

Expression (2) was derived for a thin-coated rod, thus leaving out the relation between volumes. But, as an example, for thin-walled cross-sections of bridge girders of overhead cranes, the relation of $V / V_{A}$ is significant, and, therefore, a filler-induced damping effect would be quite high.

Estimating damping properties of a cantilever beam with its rectangular thin-walled cross-section filled with a certain material helps to obtain an expression for the coefficient of structural absorption $\psi_{S}$ similar to (2).

Let us denote the highest potential energy and the one dissipated during a cycle by $P_{F}$, $P_{S}, P_{B}$ and $\Delta P_{F}, \Delta P_{S}, \Delta P_{B}$ (for a filled beam, a whole structure and a steel thin-walled beam, correspondingly). Then

$$
P_{S}=P_{F}+P_{B}, \Delta P_{S}=\Delta P_{F}+\Delta P_{B}
$$

Besides, by their definition,

$$
\begin{aligned}
& \Delta P_{S}=\psi_{S} P_{S} \\
& \Delta P_{F}=\psi_{F} P_{F} \\
& \Delta P_{B}=\psi_{B} P_{B}
\end{aligned}
$$

Thus, the absorption coefficient of a steel beam with a filler is expressed as

$$
\psi_{S}=\psi_{B} \frac{P_{B}}{P_{B}+P_{F}}+\psi_{F} \frac{P_{F}}{P_{B}+P_{F}}
$$

For a cantilever beam, it takes the form of

$$
P=\frac{\varphi^{2} E J}{2 l}
$$

where $\varphi, L, J$ are rotation angle, beam length and cross-section inertia moment. Substituting it into (3) and considering that $E_{\mathrm{B}}>>E_{\mathrm{H}}$, we have 


$$
\psi_{S}=\psi_{B}+\psi_{F} \frac{E_{F} J_{F}}{E_{B} J_{B}}
$$

Thus, an energy-based approach allows to predict a slightly-increased absorption coefficient in the case of a filled beam due to the relation of $E_{F} / E_{G}$ being too small. But even low damping could be useful for certain combinations of structural properties. The results of a computational experiment on a cantilever beam of $600 \mathrm{~mm}$ in length were partially disclosed in [6].

\section{Computational experiment to assess the efficiency of damping using a foam material for a cantilever beam}

The problem of volume damping was solved by running computational experiments in Solid Works software environment [7,8], followed by the analysis in its SolidWorks Simulation add-on. The properties of a filler (foam), which are required to be set prior to an experiment, were chosen on the basis of specification sheets for a professional line of polyurethane foams from Soudafoam.

For the research, a small beam was selected. It was made from a rectangular St3sp steel pipe (Fig. 1) which had a box section and was $b=25 \mathrm{~mm}$ wide, $h=50 \mathrm{~mm}$ high and $l=600$ $\mathrm{mm}$ long. Lower frequencies of beam vibrations were determined for the walls of $s \in[0,2$; 1,5] $\mathrm{mm}$ thick.

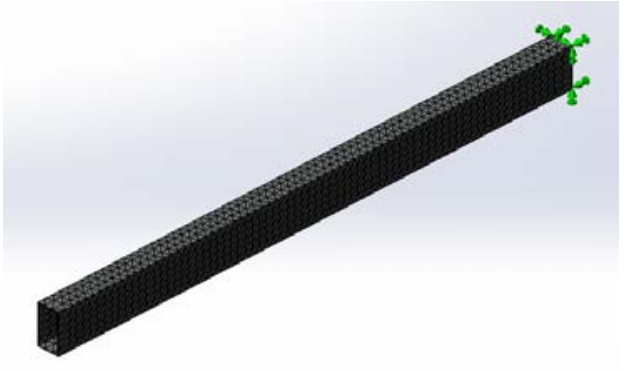

Fig. 1. Model of a beam made from finite elements.

The solution obtained for selected thicknesses recorded lower eigenfrequencies in the vertical plane for beams of various wall breadths. The results are cited in Table 1.

Table 1. Results of the finite-element analysis.

\begin{tabular}{|c|c|c|}
\hline $\begin{array}{c}\text { Wall thickness } s, \\
m m\end{array}$ & \multicolumn{2}{|c|}{ Damped natural vibrations of a beam, $\mathrm{Hz}$} \\
\cline { 2 - 3 } & Beam with a filler $\omega_{2}$ & Beam without a filler $\omega_{1}$ \\
\hline 0,2 & 136,66 & 139,560 \\
\hline 0,4 & 138,77 & 140,240 \\
\hline 0,6 & 139,96 & 140,940 \\
\hline 0,8 & 140,87 & 141,630 \\
\hline 1 & 141,71 & 142,320 \\
\hline 1,2 & 142,50 & 143,000 \\
\hline 1,5 & 143,63 & 144,030 \\
\hline
\end{tabular}


The research in [9] cites an expression for determining a logarithmical decrement of natural frequencies $\delta_{2}$ for a filled beam and known frequencies $\omega_{1}$ and $\omega_{2}$, and a decrement $\delta_{1}$ for an unfilled beam

$$
\delta_{2}=\frac{1}{\omega_{2}} \sqrt{\left(\delta_{1} \omega_{1}\right)^{2}+\omega_{1}^{2}-\omega_{2}^{2}}
$$

The logarithmical decrement for a metal structure could be assumed as 0,05 if no data is available which would prove otherwise. Fig. 2 shows how the decrement $\delta_{2}$ changes when it is calculated using expression (5) with an unchanged $\delta_{1}=0,05$.

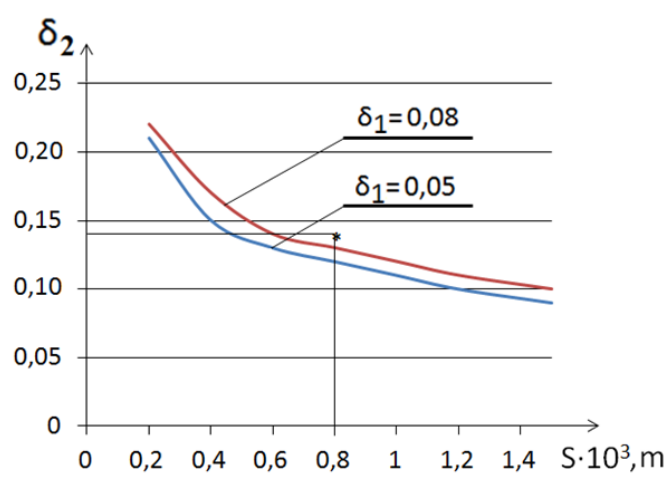

Fig. 2. Relation between logarithmical decrement $\delta_{2}$ and wall thickness s for a filled beam.

The graph above manifests a significantly-increased efficiency of volume damping while the wall thickness of the beam cross-section decreases. But only an experiment could provide a valid conclusion on the basis of its results.

\section{Testing on a cantilever beam}

Since the results cited could not have allowed to unambiguously make a conclusion on the abilities of a foam material to damp free damped vibrations of a metal structure, they were experimentally validated.

The goal of testing accompanied by plotting vibrograms for unfilled and foam-filled beams was to validate the predictions made by the computational experiment. The object of the testing was a cantilever beam with a wall of $s=0,8 \mathrm{~mm}$ thick and its other dimensions remaining the same as specified for the calculations described above.

The equipment and means of the experiment aimed to preliminarily assess the efficiency of volume damping were chosen on the condition of minimal costs needed to run it. To record vibrograms, tensometric sensors [10], oscillographs with optical systems [11] or speed cams are usually utilized. The available means dictated the usage of a high-speed cam in a Samsung S9 smart phone, which would allow to shoot 960 frames per second, thus giving an error of about $10 \%$ while plotting an oscillogram. 
An assembled test bed (Fig. 3) consists of a rectangular-section steel pipe 1, a workbench 2, where a support is mounted to clamp the pipe, a movement limiter 3 with a gap $\Delta$, a loading unit 4 and the recording cam.

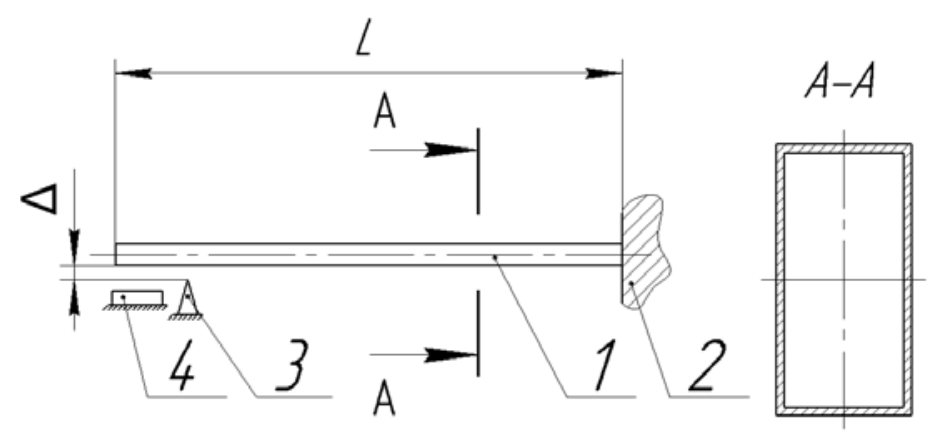

Fig. 3. Assembled test bed.

The tests were conducted in a technical diagnostics laboratory at the Institute of Engineering Science of the Ural Branch of the Russian Academy of Sciences. A TREND polyurethane foam from KUDO was used as a filler [12] after two weeks of its solidification.

\section{Discussion of the results}

Experimental vibrograms of elastic damped vibrations in the vertical plane of unfilled and foam-filled beams are given in Fig. 4. Only an envelope of vibrations is shown for the unfilled beam, thus allowing to visually demonstrate a damping effect of the foam material.

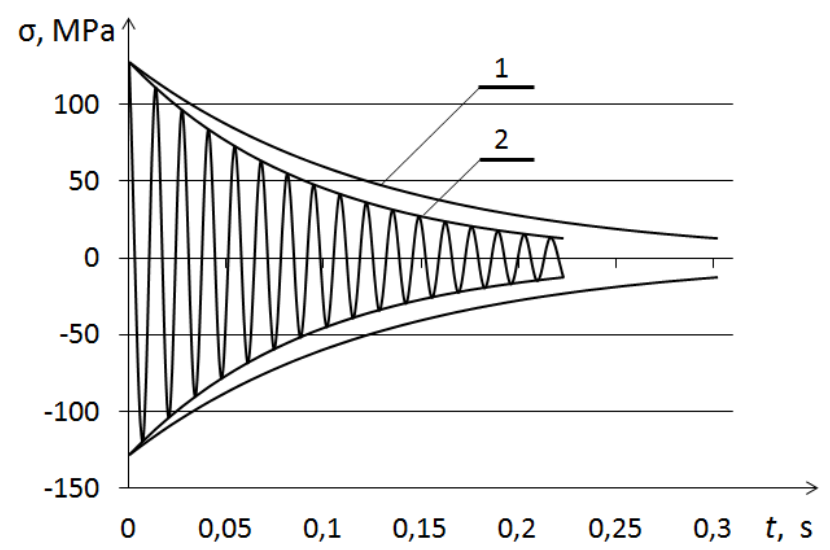

Fig. 4. Vibrograms for unfilled and filled beams.

The logarithmical decrement $\delta=\ln \left(A_{V} / A_{V+1}\right)$, determined by two neighboring amplitudes, was calculated for the unfilled beam $-\delta_{1}=0,08$. The number of significant vibration cycles decreased from 29 to 16.

Compare a curve 2 shown in Fig. 2 and plotted from (5) with an observation decrement of $\delta_{1}=0,08$. Also, an asterisk there denotes an experimental decrement for a foam-filled beam with a wall of $s=0,8 \mathrm{~mm}$ thick under vibrations in the vertical plane: $\delta_{F V}=\delta_{2}=$ 0,14 . The decrement obtained from the vibrogram is close to 0,13 - the result of calculation using (5) and frequency data in Table 1 for $s=0,8 \mathrm{~mm}$. 


\section{Conclusion}

The problem of reducing the number of free vibrations of higher-mass metal structures utilized in technological processes which require accurate positioning could have a relatively simple and reliable solution. The results of testing conducted on beams while plotting vibrograms demonstrated that volume damping could be quite efficient when it comes to damping free vibrations of a metal structure under bending. It is suggested to use expression (5) and calculation data on eigenfrequencies similar to the ones cited in Table 1 to assess the results. The obtained experimental results provide enough ground to make a conclusion on the validity of the calculations carried out earlier on damping the bridge girder of an overhead crane. The next stage of the research on the effect of volume damping would relate to selection of a foam material and determination of its mechanical and adhesive properties under operational loads.

\section{References}

1. V.T. Troshhenko, L.A. Sosnovskiy, Fatigue Strength of Metals and Alloys (Naukova Dumka, Kiev, 1987)

2. Y.G. Panovko, Internal Friction from Vibrations of Elastic Systems (Fizmatgiz, Moscow, 1960)

3. Y.G. Panovko, Basics of Applied Theory of Elastic Vibrations (State Science and Technology Publisher of Engineering Literature, Moscow, 1957)

4. V.N. Kobelev, L.M. Kovarskiy, S.I. Timofeev, Design of Three-Layer Structures (Mashinostroenie, Moscow, 1984)

5. K.V. Frolov (Ed.), Shock and Vibration Protection, vol. 6 in: V.N. Chelomey (Ed.), Vibrations in Machinery, 6 vols. (Mashinostroenie, Moscow, 1981)

6. V.I. Mironov, O.A. Lukashuk, D.A. Ogorelkov, J. MATEC Web Conf., 129 (2017) 01024

7. A.A. Aliamovskiy, A.A. Sobachkin, E.V. Odintsova, et al., Computer-Aided Design in SolidWorks for Engineering Purposes (BHV-Peterburg, Saint Petersburg, 2005)

8. K.A. Basov, ANSYS: Reference Book (DMK Press, Moscow, 2005)

9. V.I. Mironov, O.A. Lukashuk, D.A. Ogorelkov, J. MATEC Web Conf., 253 (2019) 01004

10. E.V. Hromov, O.V. Hromov, I.V. Hromov, J. Fundamental and Applied Problems of Machinery and Technology, 5, pp. 24-28 (2015)

11. I.S. Kamantsev, A.P. Vladimirov, E.M. Borodin, J. Tambov University Review, 4-2, 1881-1882 (2013)

12. TREND WINDOW 60 | TU 2254-055-18738966-2012, https://kudopurfoam.ru/pdf/trend_60_window.pdf 